\title{
Virulence of Listeria monocytogenes isolated from the cheese dairy environment, other foods and clinical cases
}

\section{Correspondence \\ Luisa Brito \\ Ibrito@isa.utl.pt}

Received 2 October 2007

Accepted 11 December 2007

\author{
Elsa Neves, ${ }^{1,2}$ Ana Carla Silva, ${ }^{1}$ Sylvie M. Roche, ${ }^{3}$ Philippe Velge ${ }^{3}$ \\ and Luisa Brito ${ }^{1}$
}
${ }^{1}$ Laboratório de Microbiologia, CBAA/DBEB, Instituto Superior de Agronomia, Technical University of Lisbon, Tapada da Ajuda, 1349-017 Lisbon, Portugal
${ }^{2}$ Instituto Superior de Estudos Interculturais e Transdisciplinares (ISEIT), Campus Universitário de Almada, Instituto Piaget, Quinta da Arreinela de Cima, 2800-305 Almada, Portugal
${ }^{3}$ Institut National de la Recherche Agronomique, Infectiologie Animale et Santé Publique, UR1282, F-37380 Nouzilly, France

\begin{abstract}
The virulence potential of 51 Listeria monocytogenes isolates, including strains from cheese, cheese production environments and from human cases of listeriosis, was evaluated in this study. The isolates were used to infect HT-29 cell monolayers in an in vitro test of virulence, based on a plaque-forming assay (PFA). Fifteen selected isolates were used for subcutaneous footpad inoculation in mice and subsequent recovery of the bacterium from the spleen 3 days after inoculation. In the PFA, two isolates from milk (serovar $1 / 2 \mathrm{a}$ ) were not significantly different $(P<0.05)$ from the low-virulence strain $(442)$ used as reference. Thirty-three isolates were not significantly different $(P<0.05)$ from the virulent strain $(E G D e)$ used as reference. Nine isolates were significantly more virulent (highly virulent) than the EGDe strain and seven isolates were significantly less virulent. The nine highly virulent isolates were either from humans (four), from cheese dairy environments (two isolates of a strain were found persistently in two dairies), from cheese (one), from milk (one) and the reference strain for serovar 1/2b (CECT 936). The two milk isolates with low virulence in the PFA were found to be virulent in mice. In conclusion, all the isolates from food and food-related environments were potentially virulent or highly virulent. These results stress the risk of listeriosis associated with the consumption of cheese contaminated with L. monocytogenes, and once more emphasize the importance of good manufacturing practices (GMPs) together with sanitation standard operating procedures (SSOPs) throughout the food chain.
\end{abstract}

\section{INTRODUCTION}

Listeria monocytogenes is a foodborne pathogen for humans and animals that causes listeriosis with high levels of mortality, particularly among immunocompromised hosts. The risks associated with this pathogen are largely due to its ubiquitous nature combined with its ability to grow under environmentally stressful conditions, namely high osmolarity, reduced oxygen tension, low $\mathrm{pH}$ and refrigerator temperatures. Currently, it is recognized that the presence of L. monocytogenes in raw foods cannot be eliminated completely, although effective hygiene practices greatly reduce its incidence. Besides host immunity and the number of ingested bacteria, virulence of the strain is an important determinant in the development of illness. Virulence gene allelic analysis, ribotyping and comparative

Abbreviation: PFA, plaque-forming assay. virulence characterization have been shown to subdivide $L$. monocytogenes into three lineages. Lineage I consists of strains (flagellar antigen types b and d) that are more likely to cause human disease than isolates classified into lineages II (antigen type a or c) and III (rarely detected serovars, 4a and 4c) (Wiedmann et al., 1997). However, it is also acknowledged that the virulence of $L$. monocytogenes strains may vary within the same serovar (Brosch et al., 1993). In a previous work, the persistence of a particular pulsotype of serovar $4 \mathrm{~b}$ (lineage I) within different traditional cheese dairies producing soft ewe's cheese was reported (Leite et al., 2006).

The purpose of this work was to evaluate the virulence of $L$. monocytogenes isolated from cheese and from its production environment (including the persistent strain) by comparing it with the virulence of isolates from different origins, including human cases of listeriosis. A previously 
described in vitro test of virulence, based on a plaqueforming assay (PFA), and the enumeration of viable bacteria in spleens after the subcutaneous inoculation of mice (Roche et al., 2001), were the tests used to investigate the pathogenic potential of the isolates.

\section{METHODS}

L. monocytogenes strains and culture conditions. This study used 51 L. monocytogenes isolates. Eight of these strains were obtained from the Collection de l'Institut Pasteur [CIP $104794=$ NCTC 7973 (serovar 1/2a)], the Colleción Española de Cultivos Tipo [CECT 936 (serovar 1/2b), CECT 911 (serovar 1/2c), CECT 937 (serovar 3b), CECT 934 (=ATCC 19114) (serovar 4a) and CECT 4032 (serovar 4b)], Dr Pascale Cossart, Institut Pasteur [EGDe (serovar 1/2a)], and the Institut National de la Recherche Agronomique, Tours [442 (serovar $4 \mathrm{~d} / 4 \mathrm{e})]$. Table 1 shows the characterization of the $51 \mathrm{~L}$. monocytogenes isolates belonging to seven serovars $(1 / 2 \mathrm{a}, 1 / 2 \mathrm{~b}, 1 / 2 \mathrm{c}$, $3 \mathrm{~b}, 4 \mathrm{a}, 4 \mathrm{~b}$ and $4 \mathrm{~d} / 4 \mathrm{e})$, including strains from milk $(n=6)$, from cheese $(n=14)$, from cheese dairy environment $(n=2)$, from milking environments $(n=1)$, from delicatessen meats $(n=5)$, from food products $(n=1)$, from human cases of listeriosis $(n=18)$, from animal cases $(n=2)$ and those with uncertain origin $(n=2)$.

Strains were stored in tryptic soy broth (Biokar Diagnostics) containing $15 \%$ glycerol at $-80{ }^{\circ} \mathrm{C}$ until use. Before each virulence test, bacteria were subcultured onto TSA-YE (Biokar Diagnostics) and subsequently grown for $18 \mathrm{~h}$ at $37{ }^{\circ} \mathrm{C}$. For the evaluation of the haemolytic activity, isolated colonies were plated onto horse blood agar (HBA) [nutritive agar, overlaid with blood agar (both from Oxoid), containing $5 \%(\mathrm{v} / \mathrm{v})$ defibrinated horse blood (Probiológica)]. Cultures were incubated at $37{ }^{\circ} \mathrm{C}$ for $48 \mathrm{~h}$.

PFGE. The $19 \mathrm{~L}$. monocytogenes isolates, that were not previously pulsotyped, were characterized in this work by DNA macrorestriction analysis using PFGE in accordance with the Pulse-Net standardized protocol (Graves \& Swaminathan, 2001). Bacterial cultures were embedded in chromosomal-grade agarose (SeaKem Gold agarose; Cambrex), lysed, washed and digested in situ overnight in separate reactions with $10 \mathrm{U} \mathrm{AscI}$ (New England Biolabs) and $20 \mathrm{U}$ ApaI (Roche Diagnostics) at 37 and $30{ }^{\circ} \mathrm{C}$, respectively. Resolution of the generated DNA fragments was obtained using $1 \%$ SeaKem Gold agarose gels in $0.5 \times$ Tris/borate/EDTA buffer (Invitrogen), run at $14{ }^{\circ} \mathrm{C}$ and $6 \mathrm{~V} \mathrm{~cm}^{-1}$ with time ramped from 4 to $40 \mathrm{~s}$ over $22 \mathrm{~h}$ using a CHEF DR II System (Bio-Rad). Gels were stained with ethidium bromide at a final concentration of $10 \mu \mathrm{g} \mathrm{ml}^{-1}$ (Sigma) and pattern images were acquired with a Bio-Rad Gel Doc 2000 imaging system. The TIFF images were normalized by aligning the peaks of the sizestandard strains Salmonella enterica serovar Braenderup H9812 and/ or L. monocytogenes CLIP 77873, which were loaded on three lanes in each gel. These strains were kindly provided by the Centre National de Référence des Listeria, Institut Pasteur, France. For each restriction enzyme, if two banding patterns differed by a single band, these patterns were considered as two different pulsotypes. The isolates were numbered after comparison with the former 25 pulsotypes described by Leite et al. (2006).

\section{In vitro test of virulence}

Cell line and culture conditions. The human adenocarcinoma cell line HT-29 (ECACC 850611109) was used between passages 20 and 44. Cells were routinely grown in $75 \mathrm{~cm}^{2}$ flasks (Orange Scientifique) in complete medium D-MEM (Dulbecco's modified Eagle's medium: high glucose, with L-glutamine, without sodium pyruvate, sodium bicarbonate) (Gibco/Invitrogen) supplemented with $3.7 \mathrm{~g}$ sodium bicarbonate $1^{-1}$ (Merck), $10 \%(\mathrm{v} / \mathrm{v})$ fetal calf serum (Gibco/ Invitrogen) and $2 \mathrm{mM}$ L-glutamine (Gibco). Penicillin (100 IU $\mathrm{ml}^{-1}$ ) and streptomycin $\left(100 \mu \mathrm{g} \mathrm{ml}^{-1}\right)$ (both from Sigma) were always added to the culture medium, except for the medium used $24 \mathrm{~h}$ prior to the virulence assay. Cells were kept in a humidified atmosphere using an incubator, at $37^{\circ} \mathrm{C}$ under $5 \%(\mathrm{v} / \mathrm{v}) \mathrm{CO}_{2}$ (ShelLab).

Plaque-forming assay (PFA). PFA was performed according to Roche et al. (2001). Briefly, HT-29 cells were trypsinized and $3 \times 10^{4}$ cells were deposited per well in a 96-well tissue culture plate (Nunc). To obtain confluent monolayers, the plates were incubated for 3 days with antibiotics followed by incubation for $24 \mathrm{~h}$ without antibiotics.

Listeria strains grown overnight on TSA-YE were then suspended in complete medium to a concentration of $4 \times 10^{8}$ cell ml ${ }^{-1}$. HT-29 cell monolayers were infected with a dilution series of $10^{2}$ to $10^{7} \mathrm{~L}$. monocytogenes per well, and incubated for $2 \mathrm{~h}$ at $37^{\circ} \mathrm{C}$. The number of viable bacteria was assessed by duplicate plating of the appropriate dilutions onto TSA-YE. Duplicates were incubated at $37{ }^{\circ} \mathrm{C}$ for $24 \mathrm{~h}$ before counting colonies.

After removing the infecting bacterial suspensions, cell monolayers were incubated for $1.5 \mathrm{~h}$ with complete medium containing $100 \mu \mathrm{g}$ gentamicin $\mathrm{ml}^{-1}$ (Sigma) without penicillin/streptomycin. Each well was then covered with complete medium supplemented with $10 \mu \mathrm{g}$ gentamicin $\mathrm{ml}^{-1}$ and containing $2.5 \%(\mathrm{~m} / \mathrm{v})$ agarose (Invitrogen). Subsequently, the same liquid medium was added to the top of the agar media to prevent cell starvation. Culture plates were incubated for 24 to $48 \mathrm{~h}$ at $37{ }^{\circ} \mathrm{C}$ under $5 \%(\mathrm{v} / \mathrm{v}) \mathrm{CO}_{2}$. Enumeration of formed plaques was performed $24 \mathrm{~h}$ after bacteria were deposited on the HT29 cell monolayer, using an inverted microscope (Leica). After $48 \mathrm{~h}$ of incubation, enumeration of the plaques was confirmed.

Mouse virulence assays. Fifteen strains belonging to serovars $1 / 2 \mathrm{a}$, $1 / 2 \mathrm{~b}, 4 \mathrm{a}$ and $4 \mathrm{~b}$ were also tested for pathogenicity by subcutaneous injection $(50 \mu \mathrm{l})$ into immunocompetent 7 -week-old Swiss female mice (Charles River), basically according to Audurier et al. (1980). Mice were housed five per cage on sterilized wood shavings and provided with water and sterilized food ad libitum. For each isolate, groups of five mice were inoculated subcutaneously (s.c.) into the left hind footpad.

L. monocytogenes isolates were grown on BHIA slopes (Difco, Becton Dickinson) for $17 \mathrm{~h}$ at $37{ }^{\circ} \mathrm{C}$. Bacteria were harvested and suspended in sterile PBS ( $\mathrm{pH} \mathrm{7.3).} \mathrm{The} \mathrm{culture} \mathrm{was} \mathrm{standardized} \mathrm{turbidime-}$ trically and diluted appropriately to obtain $4 \log$ c.f.u. in $50 \mu$ for s.c. injection. The number of viable $L$. monocytogenes cells injected was verified by plating on TSA just before administration.

Three days post-inoculation, mice were sacrificed by cervical dislocation and spleens were removed aseptically. Homogenate samples were appropriately diluted in PBS and plated onto TSA. Animal handling and care conditions were consistent for all experimental runs.

Expression of the results and data analysis. For the in vitro test, the pathogenic potential of the isolates was expressed as the mean log of the number of plaques formed (for $1 \times 10^{7}$ Listeria per well) (log PFA), in duplicate, from at least two independent trials. Strains EGDe (serovar $1 / 2 \mathrm{a}$ ) and 442 (serovar $4 \mathrm{~d} / 4 \mathrm{e}$ ) were used in the PFA as references for virulent and low-virulence strains, respectively. The previously reported low virulence of strain 442 (Roche et al., 2005) was the reason for its use as a reference for low virulence.

ANOVA of the log PFA values from the plaque-forming isolates was carried out using least significant differences (LSD) post hoc multiple comparison tests by running the program Statistica, version 6 (Statsoft). 
Table 1. Characterization of the Listeria monocytogenes isolates

Pulsotypes given in bold were determined in this study; other data were taken from Leite et al. (2006). -, Not determined.

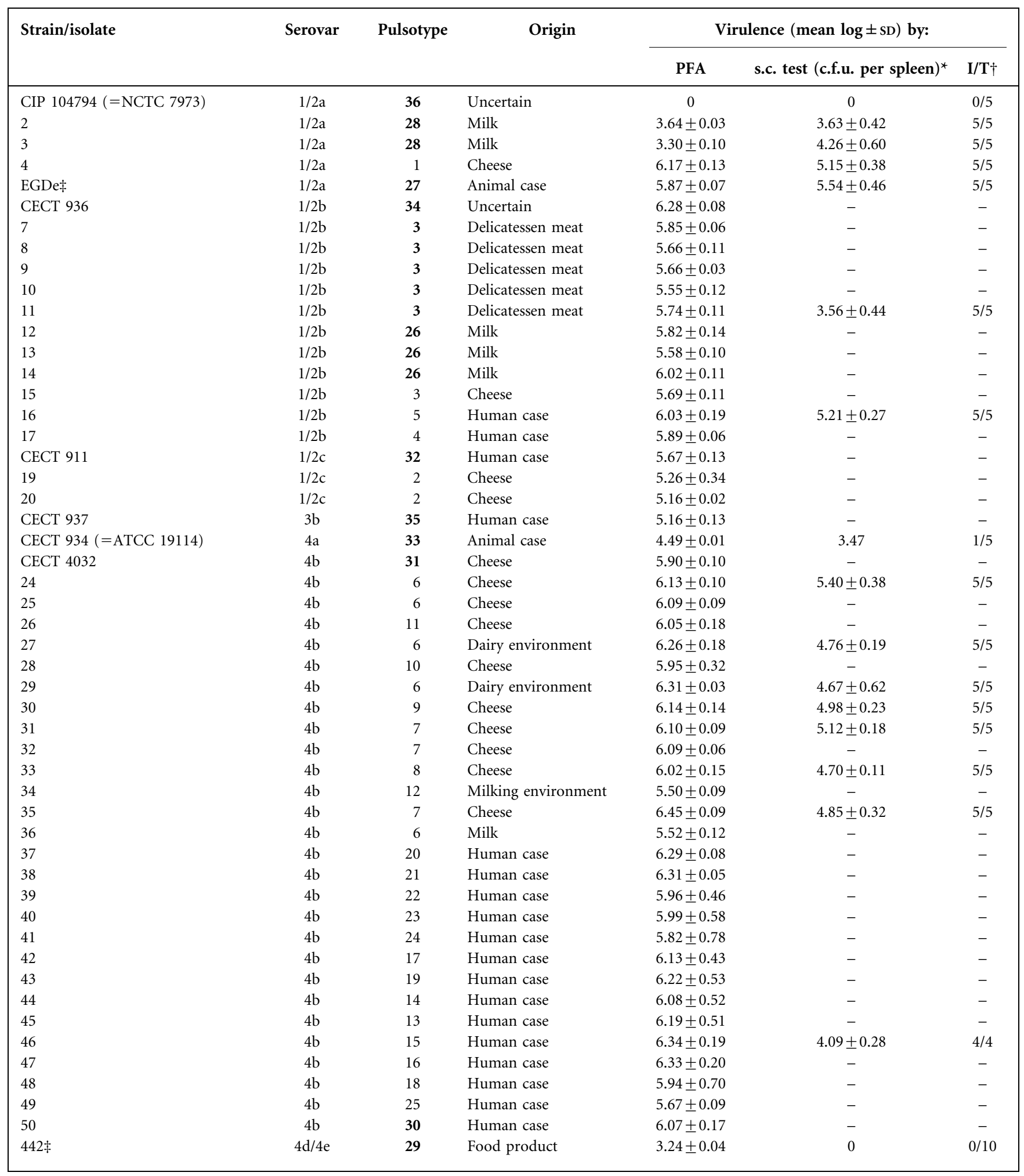

${ }^{\star} \log$ numbers of L. monocytogenes recovered in the spleens 3 days after s.c. injection into the left hind footpads of immunocompetent mice with $10^{4}$ c.f.u. in $50 \mu$ l. Values are from infected mice.

$\dagger$ Ratio of infected mice to inoculated mice in s.c. test.

$\ddagger$ Virulent (EGDe) and low-virulence (442) strains used as references for in vitro tests. Values for in vivo tests are from Roche et al. (2005). 
For the mouse virulence assay, the virulence potential of the isolates was expressed as the mean log of the number of colony-forming units ( $\log$ c.f.u.) per spleen, in addition to the ratio of infected mice to inoculated mice $(\mathrm{I} / \mathrm{T})$ in the s.c. test.

\section{RESULTS AND DISCUSSION}

\section{PFGE typing}

Nineteen out of $51 \mathrm{~L}$. monocytogenes isolates analysed were pulsotyped in this work and numbered after comparison with the former 25 pulsotypes (1-25) described by Leite et al. (2006). Following this analysis, 11 new types (26-36) were generated (Table 1). There were isolates with the same pulsotype but from different origins. This was the case for isolates 7-11 (serovar 1/2b) from delicatessen meats with the same pulsotype 3 as isolate 15 from cheese (Table 1). There was no evidence that these isolates from delicatessen meats were related. There was no association between the delicatessen meats and the cheese that were the source of these isolates. Furthermore, the five isolates showing pulsotype 6 (serovar 4b) were collected from three distinct cheese dairies: isolates 24 and 25 were from cheese produced by a particular cheese dairy; isolates 29 and 36 were collected in a second cheese dairy, from the dairy environment and milk, respectively; and isolate 27 was collected from the dairy environment of a third cheese dairy.

\section{Evaluation of virulence}

All the isolates showed haemolytic activity in HBA, but strain 442 produced weak haemolysis. In the PFA, the plaques were enumerated after $24 \mathrm{~h}$, with confirmation after $48 \mathrm{~h}$. When the plaques were large and well defined, counting after $24 \mathrm{~h}$ was usually enough. When the plaques were diffuse and small (for instance for EGDe strain), confirmation of the number of plaques was done after $48 \mathrm{~h}$. In general, the results matched and this confirmation was done routinely for confidence. The relative size of the plaques was not recorded in this study, but small as well as large plaques were found among isolates of not significantly different $(P<0.05)$ virulence.

The mean log PFA value determined in this study for the EGDe reference strain was $5.87 \pm 0.07$ (measured from 46 independent replicate tests) (Table 1 ). The value reported for this strain by Roche et al. (2001) was $6.32 \pm 0.12$ and Gudmundsdóttir et al. (2006) reported a mean value of log PFA of $4.88 \pm 0.38$ (measured 69 times). Based on these results, these authors reported cut-off values for virulence in the PFA assay of 3.34 (Roche et al., 2001) and 1.9 (Gudmundsdóttir et al., 2006). Above the cut-off value, isolates are considered virulent and below this value isolates are considered low-virulence. The values of the mean log PFA for EGDe reported by different laboratories were the reason why no cut-off value for virulence was established in this work. Instead, ANOVA of log PFA values obtained for the plaque-forming isolates was performed in order to compare their virulence with the reference strains for virulent (EGDe) and low-virulence (442) strains used in the assays.

Strain NCTC 7973 (serovar 1/2a) formed no plaques. This strain was also incapable of spleen colonization in mice. Nevertheless, Liu et al. (2003) reported strain NCTC 7973 as one of the most virulent strains tested $\left(\mathrm{LD}_{50}<10^{8}\right)$. Pine et al. (1987) reported the existence of two phenotypes for this strain, haemolytic virulent colonies and non-virulent colonies. The authors suggest that the avirulent strain is a non-haemolytic mutant of the virulent strain. However, our isolate showed haemolytic activity. This is unusual behaviour. In their review, Vázquez-Boland et al. (2001) mentioned the anomalous behaviour of NCTC 7973 as a consequence of the accumulation of several regulatory mutations. Roberts et al. (2005) proposed the classification of L. monocytogenes into four distinct virulence attenuation groups (A, B, C and D). Group D isolates were characterized by normal cytotoxicity, haemolytic activity and hlyA expression but had 'small' to 'no-plaque' phenotypes. The genetic mechanisms that cause virulence-attenuated phenotypes among group D isolates could not be determined definitively. Therefore it is possible that this isolate of NCTC 7973 can be assigned to this virulence attenuation group.

Isolates 2 and 3 (serovar 1/2a, from milk) were not significantly different $(P<0.05)$ from the low-virulence strain (442) used as reference. The remaining isolates were significantly more virulent than strain 442 . Thirty-three isolates were not significantly different $(P<0.05)$ from the virulent strain used as reference (EGDe). Nine isolates were significantly more virulent (highly virulent) than EGDe and seven isolates were significantly less virulent than EGDe. Within these seven isolates, five were also significantly more virulent than the low-virulence 442 strain. The nine highly virulent isolates were either from cheese (isolate 35), from cheese dairy environments (isolates 27 and 29), from human cases (five) and the reference strain for serovar 1/2b (CECT 936).

Fifteen isolates selected according to different levels of pathogenicity in the PFA were also tested by s.c. inoculation in mice. After inoculation of five mice with strain ATCC 19114, it was possible to recover $L$. monocytogenes from the spleen of one of the animals. This strain was previously tested by intraperitoneal (i.p.) injection and the $\mathrm{LD}_{50}$ was determined (Liu et al., 2003; Cabrita et al., 2004). The high values of $\mathrm{LD}_{50}$ obtained by these authors ( 3 logs higher than the $\mathrm{LD}_{50}$ values for the virulent strains tested, in each study) confirm the present results from the in vivo tests. Results from Liu et al. (2003) indicate the absence in strain ATCC 19114 of eight potential virulence genes (including L. monocytogenes transcriptional regulator and internalin genes). Notwithstanding, in the PFA assay this strain, although significantly less virulent than EGDe, was also significantly more virulent $(P<0.05)$ than the low-virulence reference strain 
442. Isolates 2 and 3 (serovar $1 / 2 \mathrm{a}$ ), which showed low virulence in the PFA assays, were virulent in the s.c. inoculation of mice. These two isolates were also previously characterized as virulent, following i.p. injection in mice (Cabrita et al., 2004). The agreement between a PFA with HT-29 cells and infection of immunocompetent mice was previously showed by Roche et al. (2001) and confirmed in this study for the majority of the strains tested. The discrepancy between the two models probably occurs with specific isolates. Therefore, the combination of different tests allows a better characterization of the pathogenic potential of the isolates.

In conclusion, all the isolates from food and food-related environments were potentially virulent or highly virulent. The nine highly virulent isolates (by PFA) were either from humans, from cheese or from cheese dairy environments. Of particular concern was the fact that three isolates $(24,27$ and 29) of a strain from serovar $4 \mathrm{~b}$ (pulsotype 6), found persistently in cheese and in the environments of two different cheese dairies (Leite et al., 2006), were virulent or highly virulent in the in vivo and in vitro test, respectively. These results stress the risk of listeriosis associated with the consumption of cheese contaminated with L. monocytogenes.

The implementation and maintenance of good manufacturing practices (GMPs) together with sanitation standard operating procedures (SSOPs) all along the food chain will contribute to the effective eradication of persistent strains of this pathogenic bacterium.

\section{ACKNOWLEDGEMENTS}

The authors thank Dr Christine Jacquet from Laboratoire des Listeria, Centre National de Référence des Listeria, Institut Pasteur, Paris, for allowing E. N. to stay at the Laboratory, and the members of her team for having provided the technical knowledge regarding the PFGE typing of the strains. The financial support of the Fundação para a Ciência e Tecnologia (FCT) (project POCI/SAU-ESP/56243/2004 and $\mathrm{PhD}$ grant SFRH/BD/17914/2004 to E. N.) is gratefully acknowledged.

\section{REFERENCES}

Audurier, A., Pardon, P., Marly, J. \& Lantier, F. (1980). Experimental infection of mice with Listeria monocytogenes and L. innocua. Ann Microbiol (Paris) 131B, 47-57.
Brosch, R., Catimel, B., Milton, G., Buchrieser, C., Vindel, E. \& Rocourt, J. (1993). Virulence heterogeneity of Listeria monocytogenes strains from various sources (food, human, animal) in immunocompetent mice and its associated typing characteristics. J Food Prot 56, 296-301.

Cabrita, P., Correia, S., Ferreira-Dias, S. \& Brito, L. (2004). Genetic characterization of Listeria monocytogenes food isolates and pathogenic potential within serovars $1 / 2 \mathrm{a}$ and 1/2b. Syst Appl Microbiol 27, 454-461.

Graves, L. M. \& Swaminathan, B. (2001). PulseNet standardized protocol for subtyping Listeria monocytogenes by macrorestriction and pulsed-field gel electrophoresis. Int J Food Microbiol 65, 55-62.

Gudmundsdóttir, S., Roche, S. M., Kristinsson, K. G. \& Kristjánsson, M. (2006). Virulence of Listeria monocytogenes from humans and smoked salmon, peeled shrimp, and their processing environments. J Food Prot 69, 2157-2160.

Leite, P., Rodrigues, R., Ferreira, M. A. S. S., Ribeiro, G., Jacquet, C., Martin, P. \& Brito, L. (2006). Comparative characterization of Listeria monocytogenes isolated from Portuguese farmhouse ewe's cheese and from humans. Int J Food Microbiol 106, 111-121.

Liu, D., Ainsworth, A. J. \& Austin, F. W. (2003). Characterization of virulent and avirulent Listeria monocytogenes strains by PCR amplification of putative transcriptional regulator and internalin genes. J Med Microbiol 52, 1065-1070.

Pine, L., Weaver, R. E., Carlone, G. M., Pienta, P. A., Rocourt, J., Goebel, W., Kathariou, S., Bibb, W. F. \& Malcolm, G. B. (1987). Listeria monocytogenes ATCC 35152 and NCTC 7973 contain a nonhemolytic, nonvirulent variant. J Clin Microbiol 25, 2247-2251.

Roberts, A., Chan, Y. \& Wiedmann, M. (2005). Definition of genetically distinct attenuation mechanisms in naturally virulenceattenuated Listeria monocytogenes by comparative cell culture and molecular characterization. Appl Environ Microbiol 71, 3900-3910.

Roche, S. M., Velge, P., Bottreau, E., Durier, C., Marquet-van der Mee, N. \& Pardon, P. (2001). Assessment of the virulence of Listeria monocytogenes: agreement between a plaque-forming assay with HT29 cells and infection of immunocompetent mice. Int J Food Microbiol 68, 33-44.

Roche, S. M., Gracieux, P., Milohanic, E., Albert, I., Virlogeux-Payat, I., Témoin, S., Grépinet, O., Kerouanton, A., Jacquet, C. \& other authors (2005). Investigation of specific substitutions in virulence genes characterizing phenotypic groups of low-virulence field strains of Listeria monocytogenes. Appl Environ Microbiol 71, 6039-6048.

Vázquez-Boland, J. A., Kuhn, M., Berche, P., Chakraborty, T., Domínguez-Bernal, G., Goebel, W., González-Zorn, B., Wehland, J. \& Kreft, J. (2001). Listeria pathogenesis and molecular virulence determinants. Clin Microbiol Rev 14, 584-640.

Wiedmann, M., Bruce, J. L., Keating, C., Johnson, A. E., McDonough, P. L. \& Batt, C. A. (1997). Ribotypes and virulence gene polymorphisms suggest three distinct Listeria monocytogenes lineages with differences in pathogenic potential. Infect Immun 65, 2707-2716. 